\title{
Voltage Balance Monitoring Based on Voltage's Instantaneous Space Phasor Geometrical Loci
}

\author{
A. Crăciunescu ${ }^{1}$, G. Ciumbulea ${ }^{1}$, C. Necula Dumitrică ${ }^{2}$ and M. Predescu ${ }^{3}$ \\ ${ }^{1}$ Facultatea de Inginerie Electrică \\ Universitatea Politehnica din București \\ Splaiul Independenței, 313, 060042-București, Romania \\ e-mail: aurelian.craciunescu@upb.ro, ciumbulea@yahoo.com \\ ${ }^{2}$ Facultatea de Inginerie Electrică \\ Universitatea Valahia din Târgoviște \\ B-dul Unirii, nr. 18-24, 130082-Târgoviște, Romania \\ e-mail: catalinan2003@yahoo.com \\ ${ }^{3}$ Aeolus Energy International SRL \\ Str. Nicolae Teclu, nr. 46-48, 032368-București, Romania \\ e-mail: predescu@consultant-res.ro
}

\begin{abstract}
The paper introduces a new approach for voltage balance monitoring based on the computer-aided assessment of the network Instantaneous Voltage's Space Phasor (IVSP). Between the voltage unbalances severities and the IVSP modulus ripples or the IVSP angular speed ripples are identified the appropriate correspondences which are used for voltage unbalance quantifications. The investigative studies of this new voltage unbalance approach are made on a simulated network in Simplorer programming medium.
\end{abstract}

\section{Key words}

Voltage unbalance, voltage's space phasor, phasemodulus diagrams, voltage's space phasor angular speed.

\section{Introduction}

Voltage unbalance is a serious power quality problem, mainly affecting low-voltage distribution systems, for instance encountered in office buildings with abundant PCs and lighting. However, it can be quantified in a relatively simple manner by using the instantaneous voltage's space phasor resulting in parameters that can be compared to standardized values. In technical literature can be found several techniques for voltage balance monitoring based on various unbalance factor definitions: the true factor (given by symmetrical components approach), the NEMA voltage unbalance definition, the IEEE voltage unbalance definitions or the CIGRE voltage unbalance definition $[1 \div 9]$.

The present paper introduces a new approach for voltage unbalance assessment based on the computer-aided monitoring of the IVSP in polar coordinates (phasemodulus) considered as a representative voltage unbalance factor. By using a simulated model of a threephase distribution network with various voltage unbalance severities, both in voltage amplitude as in voltage phase, the correspondences between these voltage unbalances and the IVSP modulus ripples and also with IVSP angular speed ripples are identified.

\section{The IVSP's phase-modulus approach}

As a function of the line phase voltages $v_{\mathrm{a}}, v_{\mathrm{b}}$, and $v_{\mathrm{c}}$ of a three-phase distribution system with isolated neutral point, its IVSP is given by the following equation:

$$
\vec{v}=v_{a}+v_{b} e^{j 120}+v_{c} e^{j 240}
$$

This can be written in Cartesian coordinates as:

$$
\vec{v}=v_{\alpha}+j v_{\beta}
$$

where the real and the imaginary components are:

$$
\begin{aligned}
& v_{\alpha}=v_{a}-\frac{1}{2} v_{b}-\frac{1}{2} v_{c} \\
& v_{\beta}=\frac{\sqrt{3}}{2} v_{b}-\frac{\sqrt{3}}{2} v_{c}
\end{aligned}
$$

In polar coordinates, the IVSP is:

$$
\vec{v}=m \cdot e^{j \varphi}
$$


where the modulus $m$ and the phase $\varphi$ are given by the following equalities

$$
\begin{aligned}
& m=\sqrt{v_{\alpha}^{2}+v_{\beta}^{2}} \\
& \varphi=\arccos \frac{v_{\alpha}}{m}
\end{aligned}
$$

The Simplorer simulation scheme of the three-phase distribution system with computer-aided monitoring of the line phase voltage IVSP according to the equations (3) and (5) is shown in Figure 1.

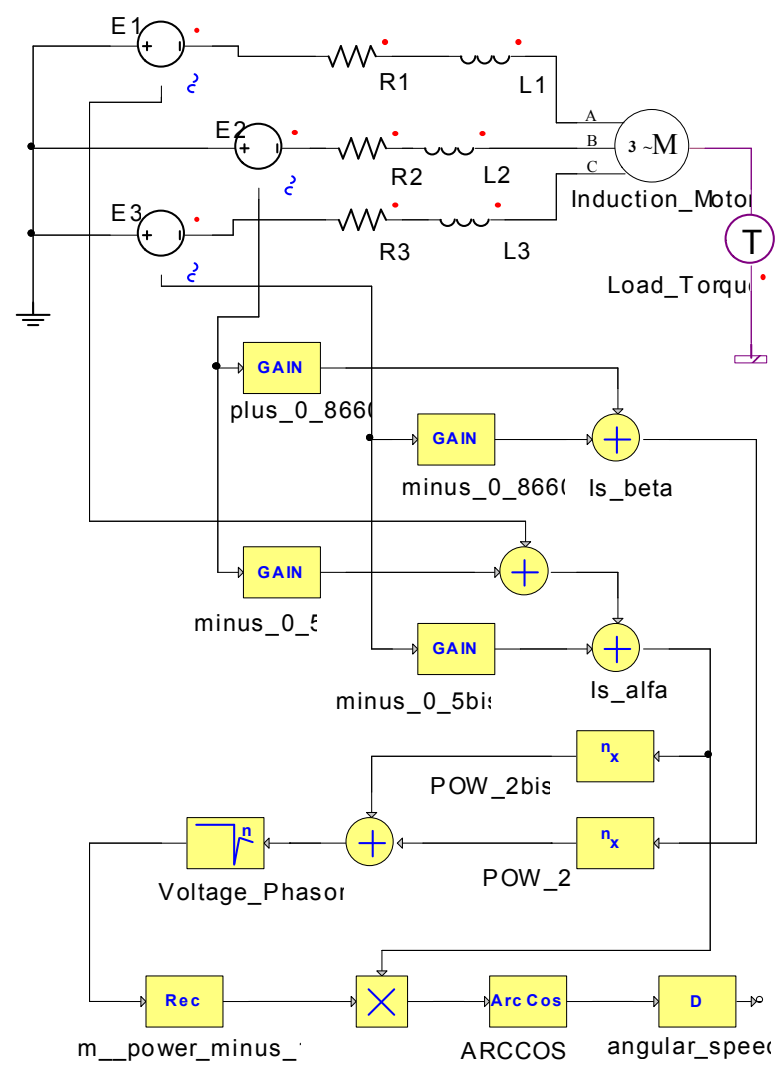

Fig. 1. The Simplorer simulation scheme of the three-phase distribution system with the monitoring of the IVSP modulus and angular speed

\section{Simulation illustrative results}

Some illustrative results concerning correspondences between various voltage unbalances and IVSP modulus ripple or IVSP angular speed ripple are shown in Tables $1,2,3$, and 4 .

In Table 1 it is shown, for three small voltage amplitude unbalances, caused by decreasing of the amplitude of voltage $v_{\mathrm{a}}$ of the phase " $a$ ", the IVSP modulus time variation, the IVSP angular speed variation and IVSP phase-modulus diagram.

In Table 2 it is shown, for the same values of the voltage amplitude unbalances, caused now by decreasing of the amplitudes of the phase " $b$ " or of the phase " $c$ " voltages $\left(v_{\mathrm{b}}\right.$ or $\left.v_{\mathrm{c}}\right)$, the diagrams of the IVSP modulus time variation, the IVSP angular speed variation and IVSP phase-modulus. It is interesting to note that the shapes of these diagrams are different and therefore it is possible to use this as a sign for the voltage amplitude unbalance localization.

In Table 3 it is shown, for three small phase voltage unbalances, caused by increasing of the phase $\varphi$ of the voltage $v_{\mathrm{a}}$, the IVSP modulus time variation, the IVSP angular speed variation and IVSP phase-modulus diagram.

In Table 4 it is shown, for the same values of the phase voltage unbalances, caused by increasing of the phase $\varphi$ of the voltages $v_{\mathrm{b}}$ or $\mathrm{v}_{\mathrm{c}}$, the IVSP modulus time variation, the IVSP angular speed variation and IVSP phasemodulus diagram. Also in this case, of the shapes of the IVSP diagrams, it is possible to localize the voltage phase unbalance.

\section{Conclusion}

The IVSP diagrams in polar coordinates: modulus time diagram, phasor angular speed time diagram and phase modulus diagram could be used as tools for amplitude or phase voltage unbalance severities evaluation. The accuracy of this tool is appropriate for the practical requirements. A comparison with other voltage unbalance evaluation techniques will be developed in a future work.

\section{References}

[1] M. H. J. Bollen, "Definition of Voltage Unbalance", IEEE Power Engineering Review, November 2002, pp. 49-50.

[2] A. Campos, G. Joos, P. D. Ziogas and J. F. Lindsay, "Analysis and Design of a Series Voltage Unbalance Compensator Based on a Three-Phase VSI Operating With Unbalanced Switching Functions", IEEE Trans. On Power Electronics, vol. 9, no. 3, May 1994, pp. 269-274.

[3] Annette von Jouanne and Basudeb (Ben) Banerjee, "Assessment of Voltage Unbalance", IEEE Trans. On Power Delivery, vol. 16, no. 4, Oct. 2001, pp. 782-790.

[4] J. Barros, R. I. Diego and M. De Apraiz, "A virtual instrument for the measurement of voltage unbalance in power systems", Proc. of the 2008 Int. Conf. on Electrical Machines ICEM 2008, 6-9 Sept. 2008, Vilamoura, paper ID 782.

[5] P. G. Kini, R. C. Bansal and R. S. Aithal, "A Novel Approach Toward Interpretation and Application of Voltage Unbalance Factor", IEEE Trans. On Industrial Electronics, vol. 54, no. 4, Aug. 2007, pp. 2315-2322.

[6] M. de Oliveira, A.L. Ferreira Filho and D. de Oliveira, "Voltage Unbalance Monitoring in CEB Systems", Proc. of the 10th Int. Conf. on Harmonics and Quality of Power, 2002, vol. 1, pp.214-219.

[7] P. Pillay and M. Manyage, "Definition of Voltage Unbalance", IEEE Power Engineering Review, May 2001, pp. 50-51.

[8] T. E. Seiphetlho and A. P. J. Rens, "On the assessment of Voltage Unbalance", Proc. of 14th Int. Conf. on Harmonics and Quality of Power, 26-29 Sept. 2010, Bergamo, Italy.

[9] A. K. Singh, G. K. Singh and R. Mitra, „Some Observations on Definitions of Voltage Unbalance", Proc. of 39th North American Power Symposium, 30 Sept.-2 Oct. 2007, pp. 473-479. 
Table 1: Voltage unbalance caused by decreasing of amplitude of $V_{a}$

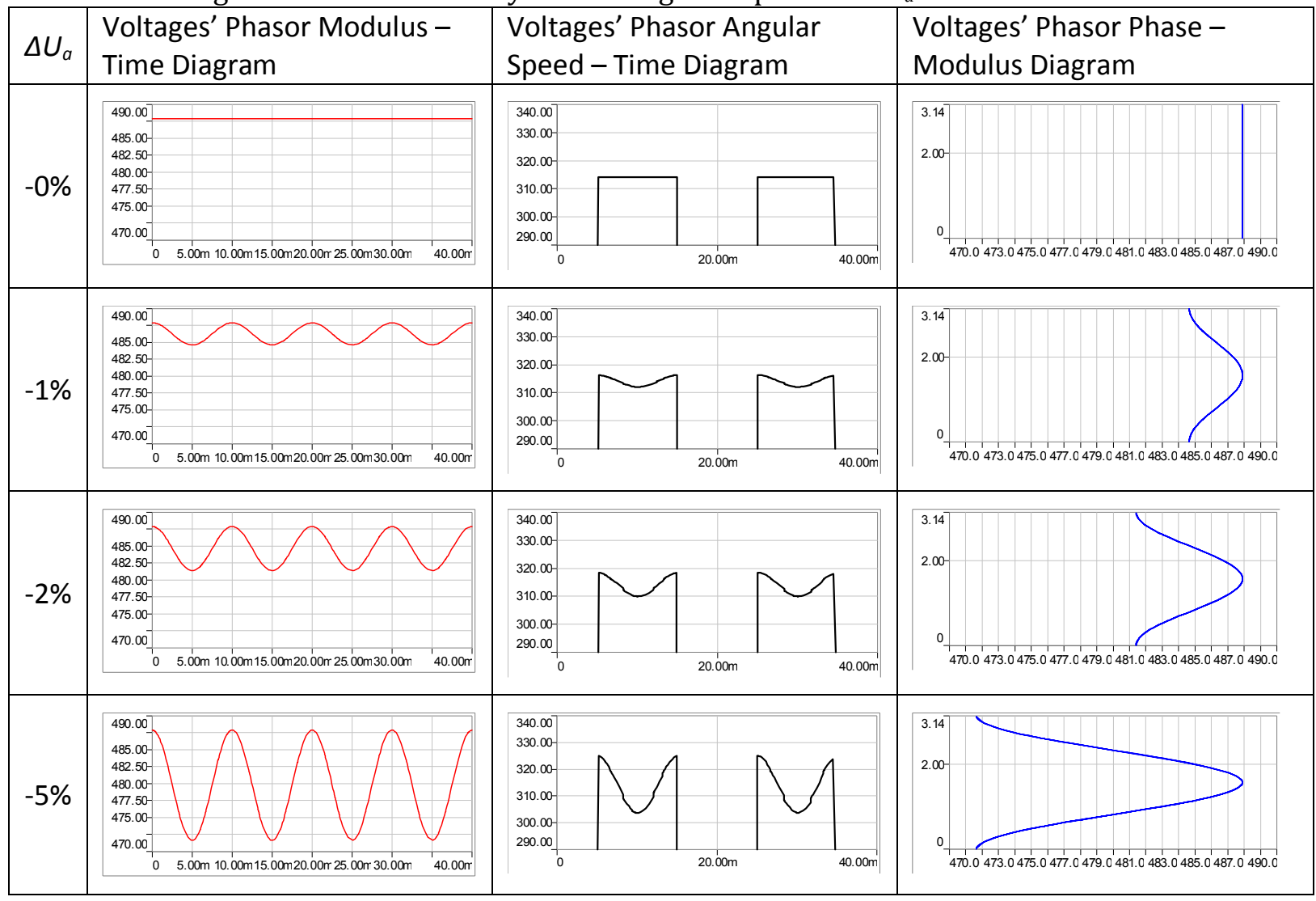

Table 2: Voltage unbalance caused by decreasing of amplitude of $V_{b}$ or $V_{c}$

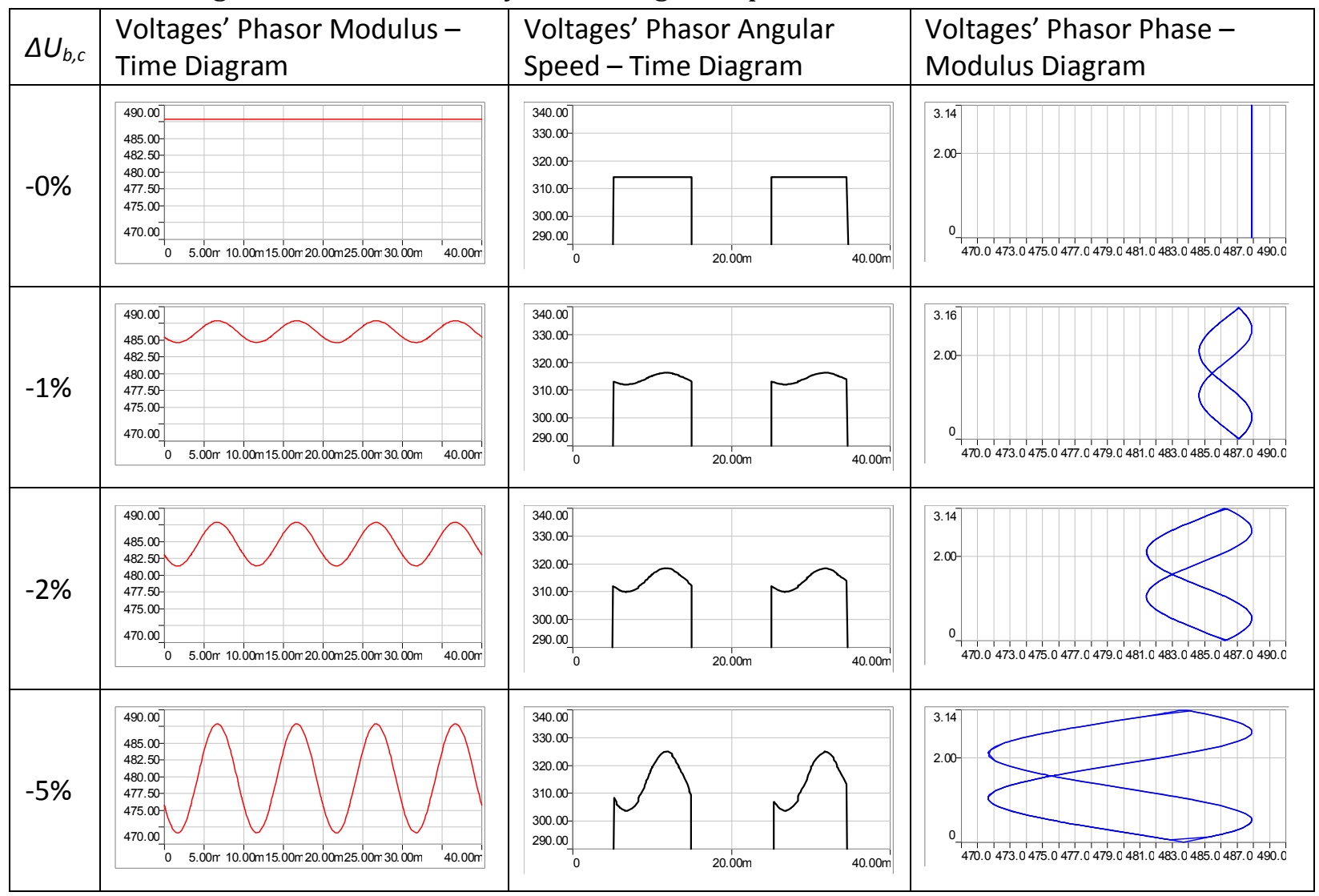


Table 3: Voltage unbalance caused by decreasing of phase angle of $V_{a}$

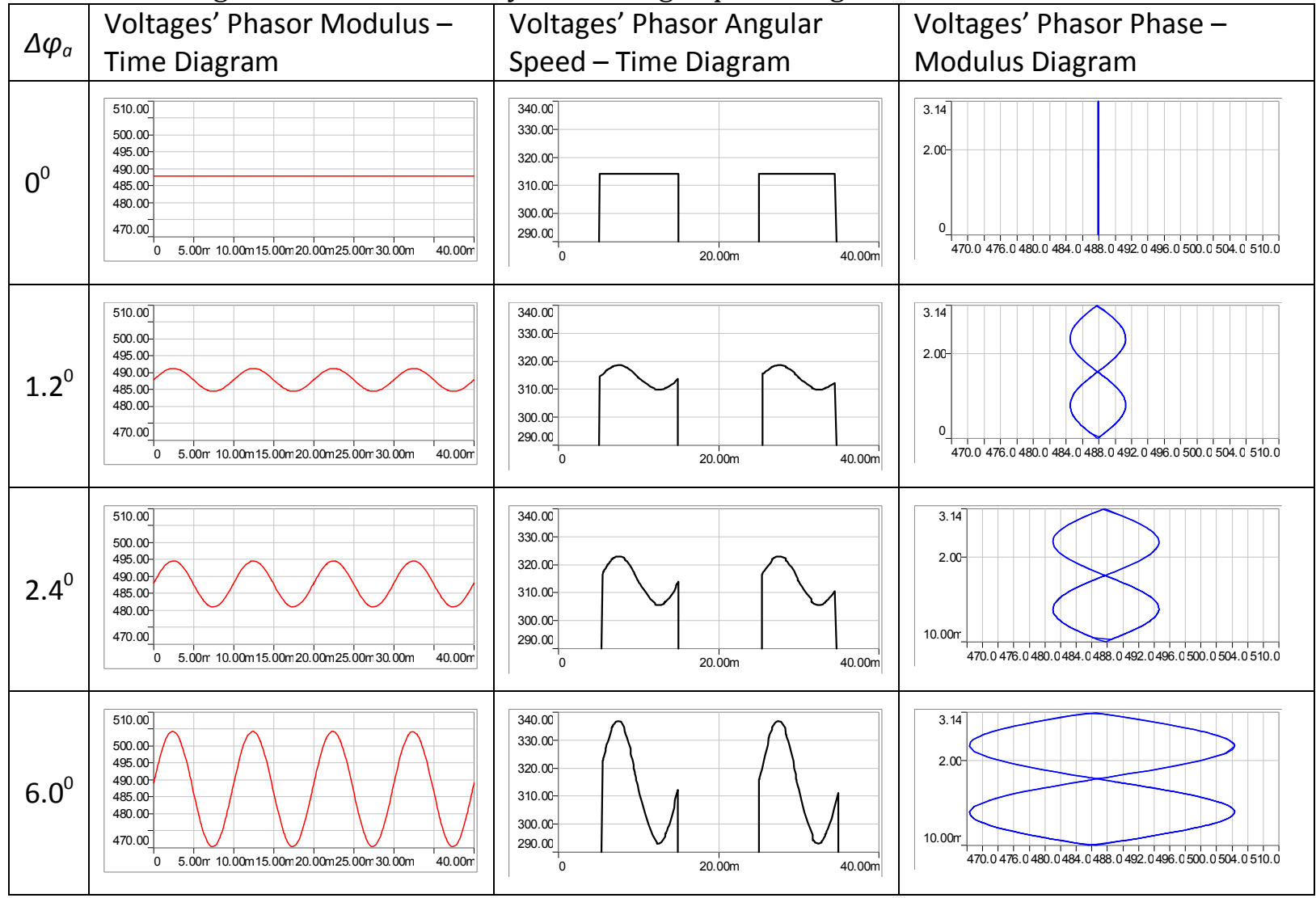

Table 4: Voltage unbalance caused by decreasing of phase angle of $V_{b}$ or $V_{c}$

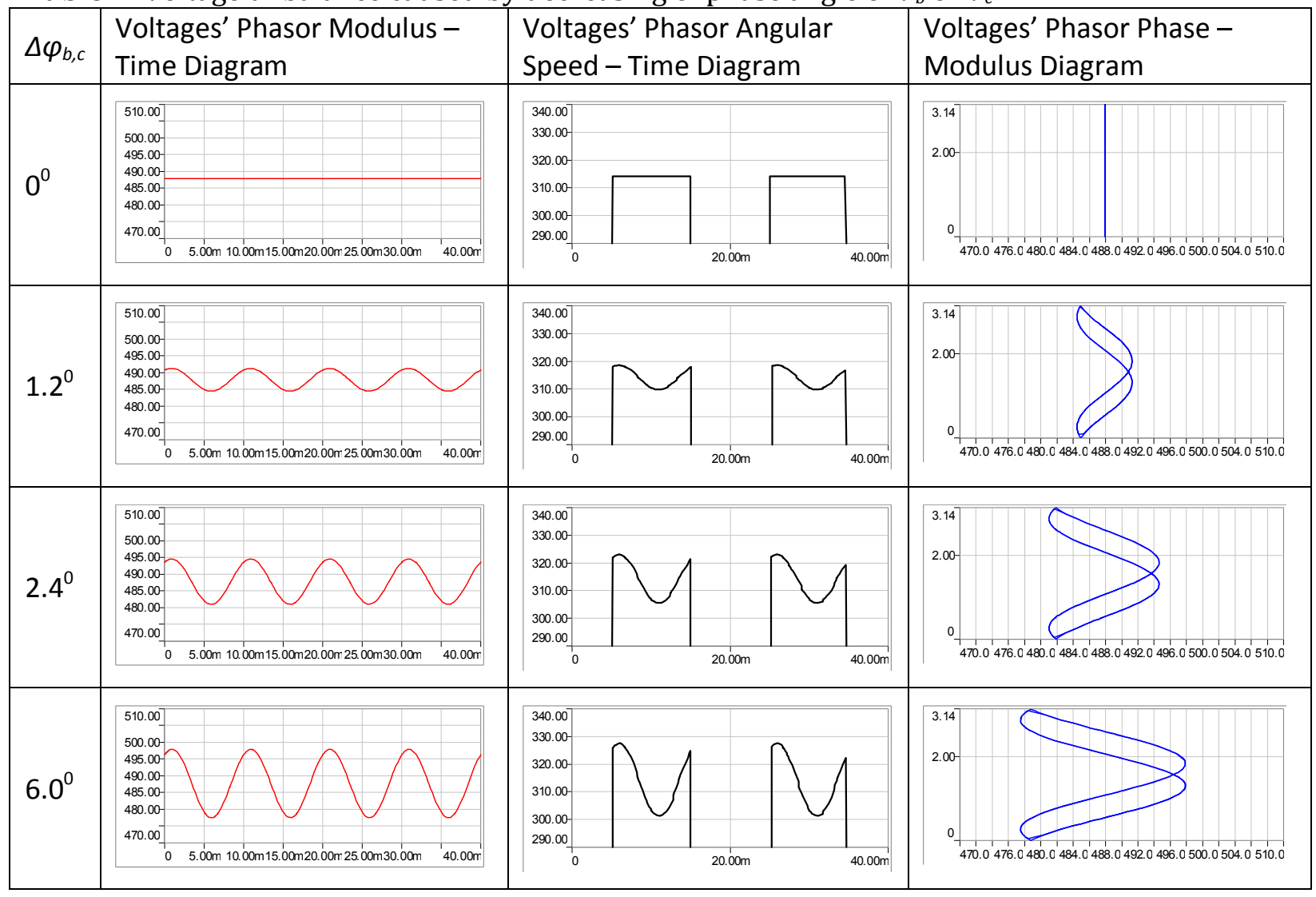

